\title{
Lie ideals and centralizing generalized derivations of rings with involution
}

\author{
Lahcen Oukhtite
}

Received: 22 September 2009 / Accepted: 8 September 2010 / Published online: 19 June 2011

C The Author(s) 2011. This article is published with open access at Springerlink.com

\begin{abstract}
A classical result of Posner states that the existence of a nonzero centralizing derivation on a prime ring forces the ring to be commutative. In this paper we extend the posner's result to the case of generalized derivations centralizing on Lie ideals of rings with involution.
\end{abstract}

Keywords Rings with involution $\cdot$ Generalized derivations

Mathematics Subject Classification (2000) $\quad$ 16W10 $\cdot 16 \mathrm{~W} 25 \cdot 16 \mathrm{~N} 60$

\section{Introduction}

Throughout $R$ will represent an associative ring with center $Z(R)$. For any $x, y \in R$, the symbol $[x, y]$ stands for the commutator $x y-y x$ and we will make use of the following basic commutator identities without any specific mention: $[x, y z]=y[x, z]+$ $[x, y] z,[x y, z]=x[y, z]+[x, z] y . R$ is 2 -torsion free if $2 x=0$ yields $x=0$. We recall that $R$ is prime if $a R b=0$ implies $a=0$ or $b=0$.

A ring with involution $(R, *)$ is $*$-prime if $a R b=a R b^{*}=0$ yields $a=0$ or $b=0$. Note that every prime ring having an involution $*$ is $*$-prime but the converse is in general not true. For example, if $R^{o}$ denotes the opposite ring of a prime ring $R$, then $R \times R^{o}$ equipped with the exchange involution $*_{e x}$, defined by $*_{e x}(x, y)=(y, x)$, is $*_{e x}$-prime but not prime. This example shows that every prime ring can be injected

L. Oukhtite $(\varangle)$

Département de Mathématiques, Faculté des Sciences et Techniques,

Groupe d'Algèbre et Applications, Université Moulay Ismaïl,

B. P. 509, Boutalamine, Errachidia, Morocco

e-mail: oukhtitel@hotmail.com 
in a $*$-prime ring and from this point of view $*$-prime rings constitute a more general class of prime rings.

An additive mapping $d: R \longrightarrow R$ is called a derivation if $d(x y)=d(x) y+x d(y)$ holds for all pairs $x, y \in R$. An additive mapping $F: R \longrightarrow R$ is said to be a generalized derivation associated with a derivation $d$ if $F(x y)=F(x) y+x d(y)$ holds for all pairs $x, y \in R$. Generally we do not mention the derivation d associated with a generalized derivation $\mathrm{F}$ rather prefer to call simply a generalized derivation. An additive mapping $g: R \longrightarrow R$ is called a left multiplier if $g(x y)=g(x) y$ for all $x, y \in R$. In particular, a generalized derivation associated with the zero derivation is a left multiplier.

A mapping $f$ of $R$ into itself is called centralizing if $[f(x), x] \in Z(R)$ holds for all $x \in R$; in the special case when $[f(x), x]=0$ holds for all $x \in R$, the mapping $f$ is said to be commuting. The history of commuting and centralizing mappings goes back to 1955 when Divinsky (1955) proved that a simple artinian ring is commutative if it has a commuting nontrivial automorphism. Two years later, Posner (1957) has proved that the existence of a nonzero centralizing derivation on a prime ring forces the ring to be commutative (Posner's second theorem). Several authors have proved commutativity theorems for prime rings or semiprime rings admitting automorphisms or derivations which are centralizing or commuting on an appropriate subset of the ring (see Bell and Martindale 1987 for a partial bibliography). Recently, Oukhtite et al. generalized Posner's second theorem to rings with involution in the case of characteristic not 2 : Let $R$ be a 2-torsion free $*$-prime ring and $U$ a square closed $*$-Lie ideal. If $R$ admits a nonzero derivation d centralizing on $U$, then $U \subseteq Z(R)$ (Oukhtite et al. 2010, Theorem 1).

In the present paper we shall attempt to generalize Posner's second theorem to generalized derivations centralizing on Lie ideals in rings with involution.

Throughout, $(R, *)$ will be a 2 -torsion free ring with involution and $S a_{*}(R):=\{r \in$ $R / r^{*}= \pm r$ \} being the set of symmetric and skew symmetric elements.

\section{Lie ideals and generalized derivations}

Lemma 1 (Oukhtite and Salhi 2008, Lemma 4) If $U \nsubseteq Z(R)$ is a $*$-Lie ideal of a 2-torsion free $*$-prime ring $R$ and $a, b \in R$ such that $a U b=a^{*} U b=0$, then $a=0$ or $b=0$.

Lemma 2 (Oukhtite and Salhi 2007a, Lemma 2.3) Let $0 \neq U$ be $a *$-Lie ideal of $a$ 2-torsion free $*$-prime ring $R$. If $[U, U]=0$, then $U \subseteq Z(R)$.

Lemma 3 (Oukhtite and Salhi 2007b, Lemma 2.2) Let $R$ be a 2-torsion free *-prime ring and $U$ a nonzero $*$-Lie ideal of $R$. If $d$ is a derivation of $R$ which commutes with $*$ and satisfies $d(U)=0$, then either $d=0$ or $U \subseteq Z(R)$.

Remark One can easily verify that the condition "d commutes with $*$ " in Lemma 3 is not necessary.

We begin with the following lemma which will be used to prove our main theorem. 
Lemma 4 Let $F$ be an additive mapping which is centralizing on a square closed $*$-Lie ideal $U \nsubseteq Z(R)$. If $R$ is *-prime, then $[F(U \cap Z(R)), U]=0$.

Proof Linearizing $[F(x), x] \in Z(R)$ we obtain

$$
[F(x), y]+[F(y), x] \in Z(R) \text { for all } x, y \in U \text {. }
$$

Now if $x \in U \cap Z(R)$, then (1) yields

$$
[F(x), y] \in Z(R) \text { for all } y \in U
$$

Replacing $y$ by $2 F(x)[u, v]$ in (2) we get $F(x)[F(x),[u, v]] \in Z(R)$ and hence

$$
[u, v] F(x)[F(x),[u, v]]=F(x)[F(x),[u, v]][u, v]=F(x)[u, v][F(x),[u, v]]
$$

so that

$$
[F(x),[u, v]]^{2}=0 \text { for all } u, v \in U
$$

Since $[F(x),[u, v]] \in Z(R)$, then (3) gives

$$
[F(x),[u, v]] R[F(x),[u, v]] R[F(x),[u, v]]^{*}=0,
$$

whence it follows that $[F(x),[u, v]] R[F(x),[u, v]]^{*}=0$ or $[F(x),[u, v]]=0$. Assume that

$$
[F(x),[u, v]] R[F(x),[u, v]]^{*}=0 .
$$

From (3), one obtains according to (2) that $[F(x),[u, v]] R[F(x),[u, v]]=0$, which gives, together with the above relation, $[F(x),[u, v]]=0$. Accordingly,

$$
[F(x),[u, v]]=0 \text { for all } u, v \in U
$$

Let in the relation (5) $u$ be $2 u w$; then we have

$$
[F(x), u][w, v]+[u, v][F(x), w]=0 \text { for all } u, v, w \in U
$$

Taking $u=v$ in the (6) we obtain

$$
[F(x), u][w, u]=0 .
$$

Replacing $w$ by $w z$ in the above relation, then we have $[F(x), u] w[z, u]=0$ and therefore

$$
[F(x), u] U[z, u]=0 \text { for all } u, z \in U
$$


From (7) it follows that $[F(x), u]=0$ or $[U, u]=0$ for all $u \in U \cap S a_{*}(R)$.

Let $w \in U$, as $w-w^{*} \in U \cap S a_{*}(R)$, then $\left[F(x), w-w^{*}\right]=0$ or $\left[U, w-w^{*}\right]=0$. If, $\left[U, w-w^{*}\right]=0$, we therefore have $[z, w]=\left[z, w^{*}\right]$ for all $z \in U$ which gives, because of (7),

$$
[F(x), w] U[z, w]^{*}=0,
$$

whence it follows that $[F(x), w]=0$ or $[U, w]=0$.

Now suppose that $\left[F(x), w-w^{*}\right]=0$; then $[F(x), w]=\left[F(x), w^{*}\right]$ and replacing $u$ by $w^{*}$ in (7) we find that

$$
[F(x), w] U[z, w]^{*}=0 \text { for all } z \in U
$$

we therefore have $[F(x), w]=0$ or $[U, w]=0$. Consequently,

$$
[F(x), u]=0 \text { or }[U, u]=0 \text { for all } u \in U
$$

Let us consider $U_{1}=\{u \in U /[F(x), u]=0\}$ and $U_{2}=\{u \in U /[U, u]=0\}$; then $U_{1}$ and $U_{2}$ are additive subgroups of $U$ such that $U=U_{1} \cup U_{2}$ and therefore $U=U_{1}$ or $U=U_{2}$. Since $U \nsubseteq Z(R)$, one obtains according to Lemma 2 that $U=U_{1}$, whence it follows that $[F(x), U]=0$, which completes the proof.

Now we are ready to state the main result of this paper.

Theorem 1 Let $(R, *)$ be a 2-torsion free ring with involution and $F$ a generalized derivation centralizing on a square closed $*$-Lie ideal $U$. If $R$ is $*$-prime, then $U \subseteq Z(R)$ or $F$ is a left multiplier.

Proof Let $F$ be a generalized derivation associated with a derivation $d$ and assume that $U \nsubseteq Z(R)$. Clearly, $F$ is a left multiplier if and only if $d=0$. Suppose first that $U \cap Z(R) \neq 0$ and let $0 \neq u \in U \cap Z(R)$.

Replacing $x$ by $2 u y$ in (1) and using Lemma 4, we find that $u[d(y), y] \in Z(R)$ and thus

$$
u R[[d(y), y], r]=0 \text { for all } y \in U \text { and } r \in R .
$$

Since $u^{*} \in U \cap Z(R)$, a similar reasoning leads to

$$
u^{*} R[[d(y), y], r]=0 \text { for all } y \in U \text { and } r \in R
$$

From relations (8) and (9), it follows, according to the $*$-primeness of $R$, that $[[d(y), y], r]=0$, which leads to $[d(y), y] \in Z(R)$ for all $y \in U$, whence it follows that $d$ is centralizing on $U$. Applying Oukhtite et al. (2010), Theorem 1, we obtain $d=0$.

Assume now that $U \cap Z(R)=0$. Since $F$ is centralizing, we obtain, because of $[F(x), x] \in U$,

$$
[F(x), x]=0 \text { for all } x \in U \text {. }
$$


Linearizing (10) we find that

$$
[F(x), y]+[F(y), x]=0 \text { for all } x, y \in U
$$

Putting $2 x y$ for $x$ in (11), we arrive at

$$
x[d(y), y]+[x, y] d(y)=0 .
$$

Substituting $2 z x$ for $x$ in (12) and using (12), we find that $[z, y] x d(y)=0$. We therefore have

$$
[z, y] U d(y)=0 \text { for all } y, z \in U
$$

From the above relation, it follows, according to Lemma 1, that

$$
d(y)=0 \text { or }[y, U]=0 \text { for all } y \in U \cap S a_{*}(R) .
$$

Let $u \in U$; since $u-u^{*} \in U \cap S a_{*}(R)$, then $d\left(u-u^{*}\right)=0$ or $\left[u-u^{*}, U\right]=0$. If $\left[u-u^{*}, U\right]=0$, then $[z, u]=\left[z, u^{*}\right]$ for all $z \in U$ and (13) assures that

$$
[z, u]^{*} U d(u)=0
$$

whence it follows, applying again Lemma 1 , that $d(u)=0$ or $[u, U]=0$.

If $d\left(u-u^{*}\right)=0$; replacing $y$ by $u^{*}$ in (13) we find that $[z, u]^{*} U d(u)=0$, which leads to $d(u)=0$ or $[u, U]=0$. Consequently,

$$
d(u)=0 \text { or }[u, U]=0 \text { for all } u \in U .
$$

Set $U_{1}=\{u \in U / d(u)=0\}$ and $U_{2}=\{u \in U /[u, U]=0\}$; it is clear that $U_{1}$ and $U_{2}$ are subgroups of $U$ such that $U_{1}=U$ or $U_{2}=U$ and thus $U=U_{1}$ or $U=U_{2}$. The fact that $U \nsubseteq Z(R)$ forces, because of Lemma 2, $U=U_{1}$. We therefore have $d(U)=0$, whence it follows, according to Lemma 3, that $d=0$ which proves that $F$ is a left multiplier.

The following example proves the necessity of the *-primeness hypothesis in Theorem 1.

Example Let $S=\mathbb{R}[X] \times \mathbb{R}[X]$; if we define an addition on $S$ by componentwise and multiplication by $\left(P_{1}, P_{2}\right)\left(Q_{1}, Q_{2}\right)=\left(P_{1} Q_{2}-P_{2} Q_{1}, 0\right)$, then clearly $S$ is a ring such that $s^{2}=0$ for all $s \in S$. Moreover, $S$ is noncommutative and $s t=-t s$ for all $s, t \in S$. Let $F$ be the additive mapping defined on the ring

$$
R=\left\{\left(\begin{array}{ll}
x & 0 \\
y & x
\end{array}\right) / x, y \in S\right\} \text { by } F\left(\left(\begin{array}{ll}
x & 0 \\
y & x
\end{array}\right)\right)=\left(\begin{array}{cc}
0 & 0 \\
x-y & 0
\end{array}\right) \text {. }
$$


Clearly, $F$ is a generalized derivation associated with the nonzero derivation $d$ defined on $R$ by $d\left(\left(\begin{array}{ll}x & 0 \\ y & x\end{array}\right)\right)=\left(\begin{array}{cc}0 & 0 \\ -y & 0\end{array}\right)$.

Since $s t=-t s$ for all $s, t \in S$, it is easy to check that the map $*: R \longrightarrow R$ defined by $\left(\begin{array}{ll}x & 0 \\ y & x\end{array}\right)^{*}=\left(\begin{array}{cc}-x & 0 \\ -y & -x\end{array}\right)$ is an involution.

On the other hand, if we set $a=\left(\begin{array}{ll}0 & 0 \\ s & 0\end{array}\right) \in R$, where $s \neq 0$, then $a R a=0$ and $a R a^{*}=0$; proving that $R$ is a non $*$-prime ring.

Let $U=\left\{\left(\begin{array}{ll}0 & 0 \\ y & 0\end{array}\right) / y \in S\right\}$; it is clear that $U$ is a square closed $*$-Lie ideal of $R$. Since $[F(u), u]=0$ for all $u \in U$, the $F$ is centralizing on $U$. Moreover, if $s, t \in S$ are such that $s t \neq 0$, then $u=\left(\begin{array}{ll}0 & 0 \\ s & 0\end{array}\right) \in U$ and $r=\left(\begin{array}{ll}t & 0 \\ s & t\end{array}\right) \in R$ and $[u, r] \neq 0$, proving that $U \nsubseteq Z(R)$. Since $d \neq 0$, then $F$ is not a left multiplier. Accordingly, in Theorem 1 the hypothesis of $*$-primeness is crucial.

Corollary 1 Let $(R, *)$ be a 2-torsion free ring with involution and $F$ a centralizing generalized derivation associated with a nonzero derivation. If $R$ is $*$-prime, then $R$ is commutative.

For a 2-torsion free prime ring, the conclusion of Theorem 1 was obtained in (Rehman 2002, Theorem 3.1) with the restriction that the generalized derivation is assumed to be commuting and not centralizing in general. Application of Theorem 1 yields the following result which improves (Rehman 2002, Theorem 3.1) as follows:

Theorem 2 Let $R$ be a 2-torsion free ring and $F$ a generalized derivation centralizing on a square closed Lie ideal $U$. If $R$ is prime, then $U \subseteq Z(R)$ or $F$ is a left multiplier.

Proof Assume that $F$ is a generalized derivation associated with a derivation $d$. Let $\mathcal{F}$ be the additive mapping defined on $\mathcal{R}=R \times R^{0}$ by $\mathcal{F}(x, y)=(F(x), y)$. Clearly, $\mathcal{F}$ is a generalized derivation associated with the derivation $\mathcal{D}$ defined on $\mathcal{R}$ by $\mathcal{D}(x, y)=$ $(d(x), 0)$. Moreover, if we set $W=U \times U$, then $W$ is a square closed $*_{\text {ex }}$-Lie ideal of $\mathcal{R}$. As $F$ is centralizing on $U$, it's easy to check that $\mathcal{F}$ is centralizing on $W$. Since $\mathcal{R}$ is a $*_{\text {ex }}$-prime ring, in view of Theorem 1 we deduce that $W \subseteq Z(\mathcal{R})$ or $\mathcal{D}=0$. Accordingly, either $U \subseteq Z(R)$ or $F$ is a left multiplier.

Corollary 2 Let $R$ be a 2-torsion free prime ring. If $R$ admits a centralizing generalized derivation $F$, then $R$ is commutative or $F$ is a left multiplier.

Corollary 3 (Rehman 2002, Theorem 3.1) Let $R$ be a 2-torsion free prime ring and $U$ be a square closed Lie ideal. If $R$ admits a generalized derivation $F$ such that $[F(u), u]=0$ for all $u \in U$, then $U \subseteq Z(R)$ or $F$ is a left multiplier.

Corollary 4 (Posner's Second Theorem in char $\neq 2$ ) Let $R$ be a 2-torsion free prime ring. If $R$ admits a nonzero centralizing derivation, then $R$ is commutative. 
Open Access This article is distributed under the terms of the Creative Commons Attribution Noncommercial License which permits any noncommercial use, distribution, and reproduction in any medium, provided the original author(s) and source are credited.

\section{References}

Bell, H.E., Martindale, W.S. III: Centralizing mappings of semiprime rings. Can. Math. Bull. 30(1), 92-101 (1987)

Divinsky, N.: On commuting automorphisms of rings. Trans. Roy. Soc. Can. Sect. III. 3(49), 1922 (1955)

Oukhtite, L., Salhi, S.: Centralizing automorphisms and Jordan left derivations on $\sigma$-prime rings. Adv. Algebra 1(1), 19-26 (2008)

Oukhtite, L., Salhi, S.: Lie ideals and derivations of $\sigma$-prime rings. Int. J. Algebra 1(1), 25-30 (2007a)

Oukhtite, L., Salhi, S.: $\sigma$-Lie ideals with derivations as homomorphisms and anti-homomorphisms. Int. J. Algebra 1(5), 235-239 (2007b)

Oukhtite, L., Salhi, S., Taoufiq, L.: Commutativity conditions on derivations and Lie ideals in $\sigma$-prime rings. Beiträge Algebra Geom. 51(1), 275-282 (2010)

Posner, E.: Derivations in prime rings. Proc. Am. Math. Soc. 8, 1093-1100 (1957)

Rehman, N.: On commutativity of rings with generalized derivations. Math. J. Okayama Univ. 44, 4349 (2002) 